\title{
Neural stem cells: mechanisms and modeling
}

\author{
Jun Yao*, Yangling Mu*, Fred H. Gage ${ }^{\bowtie}$ \\ Laboratory of Genetics, The Salk Institute for Biological Studies, La Jolla, CA 92037, USA \\ $\bowtie$ Correspondence: gage@salk.edu
}

Erratum to: Protein Cell 2012, 3(4): 251-261

DOI 10.1007/s13238-012-2033-6

1. "In the adult brain, neural stem cells have been found in two major niches: the hippocampus and the olfactory bulb" should be changed to: "In the adult brain, neural stem cells have been found in two major niches: the dentate gyrus and the subventricular zone" in the first sentence in the abstract.

2. "In the olfactory bulb, type B and C cells correspond to the type 1 and 2 progenitor cells in the SGZ" should be changed to: "In the subventricular zone, type $B$ and $C$ cells correspond to the type 1 and 2 progenitor cells in the SGZ" in the legend of Fig.1B.

3. "In the adult brain, there are two NSC niches, the SGZ of the dentate gyrus and the SVZ of the olfactory bulb" Should be changed to: "In the adult brain, there are two NSC niches, the SGZ of the dentate gyrus and the SVZ of the lateral ventricles" in the second sentence in the concluding remarks.

The online version of the original article can be found at http://dx.doi.org/ 10.1007/s13238-012-2033-6 